\title{
The Second Asian Radiology Summit
}

\section{Residency training program and board examination of radiology and imaging in Asia}

\author{
Noriyuki Tomiyama $^{1} \cdot$ Kei Yamada $^{2} \cdot$ Yoshiyuki Watanabe $^{1} \cdot$ Hiroshi Honda $^{3}$
}

Published online: 5 December 2017

C Japan Radiological Society 2017

\section{Introduction}

The Second Asian Radiology Summit was successfully held in Yokohama, Japan, on 14 April 2017. It was arranged to learn about the Radiology Residency Training Programs and Board Examinations in the participating countries. The event was attended by the presidents and official representatives of radiological societies in Asia. Delegates gave a presentation on the current residency training programs in their respective countries, tackled the weaknesses and strengths of their programs, and exchanged ideas on how the areas for improvement can be addressed. It was a great opportunity to continue building friendships and intensify relationships and collaborations among Asian radiological societies.

The participating societies included the Bangladesh Society of Radiology and Imaging (BSRI), Chinese Taipei Society of Radiology (CTSR), Hong Kong College of Radiologists (HCR), Korean Society of Radiology (KSR), Mongolian Radiological Society (MRS), Myanmar Radiological Society (MRS), Nepal Radiologist Association (NRA), Radiological Society of Pakistan (RSP), Philippine College of Radiology (PCR), Radiological Society of Thailand (RST), Singapore Radiological Society (SRS), Sri Lanka College of Radiologists (SCR), and Japan Radiological Society (JRS). Figure 1 is a photo of the delegates to the Second Asian Radiology Summit.

Noriyuki Tomiyama

tomiyama@radiol.med.osaka-u.ac.jp

1 Department of Radiology Graduate School of Medicine, Osaka University, Osaka, Japan

2 Department of Radiology, Kyoto Prefectural University of Medicine, Kyoto, Japan

3 Department of Radiology, Faculty of Medicine, Kyushu University, Fukuoka, Japan
Prof. Hiroshi Honda, President of the Japan Radiological Society, graced the event chaired by Profs. Noriyuki Tomiyama and Kei Yamada.

This article is a digest of the Radiology Residency Training Programs and Board Examinations in Asia as presented by the delegates.

\section{Residency training program in radiology and imaging in Bangladesh}

The residency program in radiology and imaging in Bangladesh is administered by the Bangabandhu Sheikh Mujib Medical University (BSMMU), the only medical university in the capital city, Dhaka, and a former institute of postgraduate medical education and research. The program consists of two phases, phase A and phase B, and it takes a total of 5 years to complete.

Phase A starts with an entrance examination for which the prerequisites are: (1) a Bachelor of Medicine, Bachelor of Surgery (MBBS), (2) 1 year internship, (3) completion of 1 year after internship, and (4) Bangladesh Medical and Dental Council (BMDC) registration.

Candidates for residency in the clinical faculties have to sit for a written multiple-choice admission test based on basic medical sciences (anatomy, pharmacology, physiology, biochemistry, pathology, microbiology, etc.) and facultybased topics. The questions are selected from the subjects taught in the undergraduate medical course. Foreign candidates having equivalent qualifications for admission can appear at the examination.

The completion of phase A takes 2 years and covers basic subjects and rotational training in parent and respective departments. Three months are spent on departmental orientation, 6 months on radio physics of X-rays, CT, USG, MRI, and nuclear medicine, 6 months on clinical orientation with placement in different specialties, 3 months on 


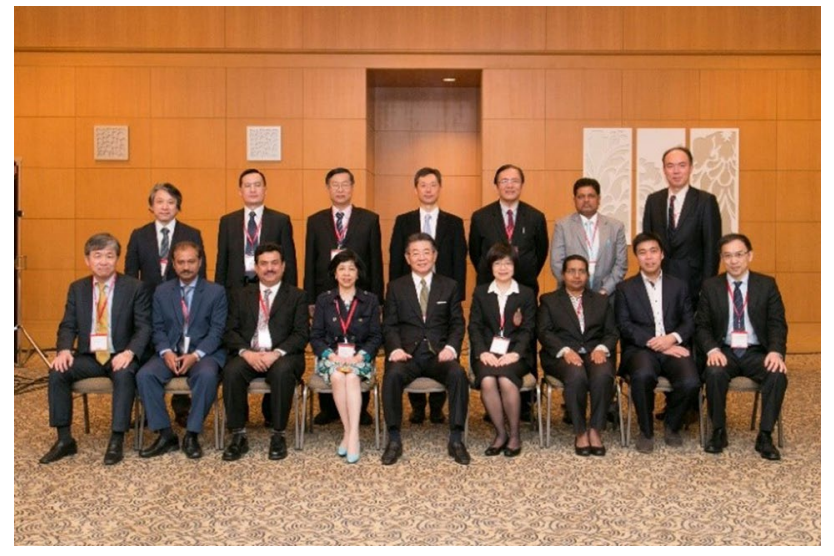

Fig. 1 Delegates at the Second Asian Radiology Summit. From left on the first row: Prof. Won Jae Lee, Dr. Khalilur Rahman, Dr. Khawaja Khurshid Ahmad, Dr. Lilian Leong, Prof. Hiroshi Honda, Dr. Juntima Euathrongchit, Dr. Shanthini Rosairo, Dr. Andrew Tan, Dr. Yeun-Chung Chang. From left to right, second row: Prof. Kei Yamada, Prof. Dagvasumberel Gonchigsuren, Dr. Kuncheng Li, Prof. Noriyuki Tomiyama, Dr. Jerome A. Gaerlan, Dr. Mukhtar Alam Ansari, and Prof. Yoshiyuki Watanabe

theoretical classes, which are arranged through negotiation with the respective departments on radio-anatomy, 3 months in the parent department, and, finally, a 3-month examination block.

Table 1 shows the contents of the phase A examination.

The second phase of residency, phase B, shown in Table 2, comprises the core theory of radiology, practical, departmental seminar, case presentation, journal club, biostatistics, research on a specific topic and thesis paper, and at least two publications in recognized journals. All of these are to be completed in 3 years.

Table 1 Contents of the phase A examination

Table 2 Contents of the phase B examination
A log book is maintained throughout the course of 5 years to monitor the program and for final assessment.

The entire program is steered by a systematic curriculum; however, having to wait for a year after internship to appear at the entrance examination is completely unnecessary. In addition, the remuneration given to the student is very low, making the chances of dropout higher. These are only a few of the challenges facing Bangladesh's residency training program.

\section{Residency training program and board examination in Taiwan}

The current status, particularly, the population of practicing radiologists in Taiwan, is only about 1300 or $2.85 \%$ of the total number of medical physicians in the country. Currently, the system of residency training in Taiwan comprises 7 years in medical school, 1 post-graduate year, residency for 4 years, and, finally, the board examination.

Starting in 2019, however, the number of years spent in medical school and post-graduate studies will change to 6 and 2 years, respectively. The residency training of 4 years will remain, covering basic medical courses as well as eight subspecialties, namely, neuro-, chest, cardiovascular, breast, GI, GU, pediatric, and interventional radiology.

There are a total of 37 hospitals in Taiwan where residents can apply for their residency training; however, only 53 residents per year are admitted for residency. This number is determined by the Resident Review Committee (RRC) of the Department of the Ministry of Health and Welfare. The resident capacity of each training hospital is determined by the committee of the Radiological Society of the Republic of China (RSROC).

\begin{tabular}{lllll}
\hline Examination type & Paper type & Subject & Total marks & Passing marks \\
\hline Written & Paper I & Radio-physics & 100 & 60 \\
Written & Paper II & Radio-anatomy & 100 & 60 \\
Oral & Paper I and II & & 100 & 60 \\
Clinical and practical paper & I and II(OSPE or objec- & 100 & 60 \\
& $\quad$ tive structured practical & & \\
Log book assessment & examination) & 100 & 75 \\
\hline
\end{tabular}

\begin{tabular}{lllll}
\hline Examination type & Paper type & Subject & Total marks & Passing marks \\
\hline Written & Paper I & General radiology & 100 & 60 \\
Written & Paper II & Special radiology & 100 & 60 \\
Oral & Paper I and II & & 100 & 60 \\
Clinical and practical paper & I and II(OSPE) & & 100 & 60 \\
Log book assessment & & & 100 & 75 \\
Thesis defense & & 100 & 60 \\
\hline
\end{tabular}




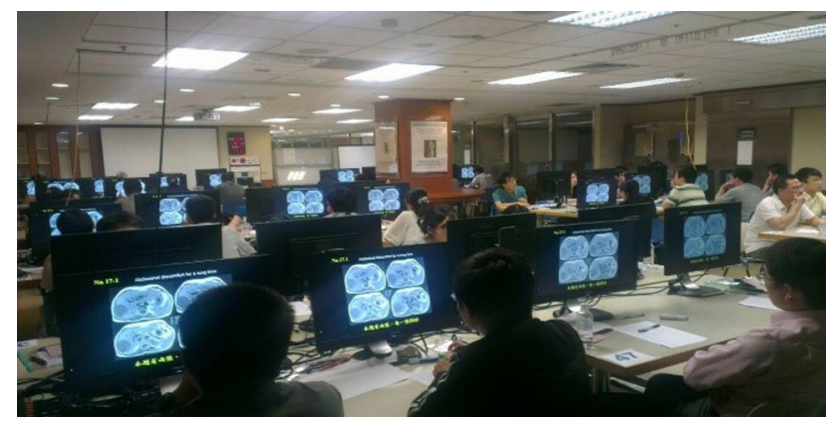

Fig. 2 Residents taking a written and film analysis test in Taiwan

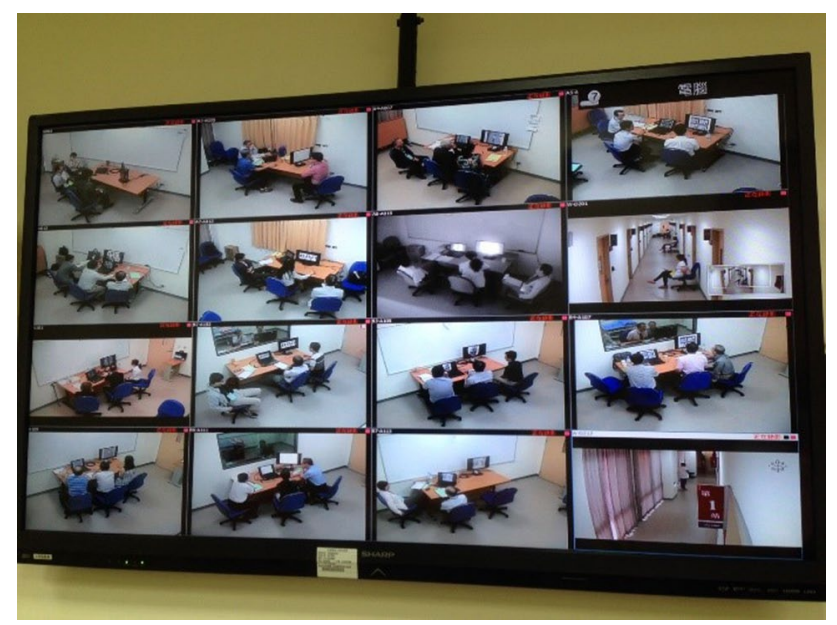

Fig. 3 Residents taking their oral examination in Taiwan

After the 4-year residency, candidates have to take the 2-day board examination. Day 1 includes a written examination and film-analyzing test (Fig. 2). Only those who pass this test can move on to day 2 for the oral examination (Fig. 3). The average pass rate for the past 10 years has been $88.5 \%$.

In general, a 4-year residency training is sufficient for fundamental requirements of becoming a board-certified radiologist. Regular on-site accreditation for the training hospitals, regular tests for residents in training for the whole country, and regular courses for medical physics will further improve the system. Additional training of 2 more years for interventional neuroradiologists, and perhaps some specific experience or case number accumulation for certain therapies such as Y-90 (yttrium-90), etc., will be great advancements as well.

\section{Residency training program and board examination in Hong Kong}

In Hong Kong, the Medical Council of Hong Kong and the Dental Council of Hong Kong are the statutory bodies established under the Medical Registration Ordinance and
Dentists Registration Ordinance. They are responsible for licensing and specialist registration of medical practitioners and dentists, respectively. Established in 1993, the Hong Kong Academy of Medicine (HKAM) has been vested with the statutory power to govern the training, qualification, and continued medical education of specialists. The trainees under the Academy Colleges must comply with the unified training requirement of a minimum of 1-year internship plus 6 years of post-graduate training.

Hong Kong College of Radiologists (HKCR) was incorporated in 1991 and is one of the 15 constituent Colleges of the Hong Kong Academy of Medicine. The HKCR encompasses three specialties, namely Radiology, Clinical Oncology, and Nuclear Medicine, and possesses the delegated statutory power to hold professional examinations for those who satisfy the requirement of supervised training and to accredit continuing medical education in these specialties. Before 1992, the training accreditation and examination for radiology in Hong Kong were conducted by The Royal College of Radiologists (RCR).

Since 1994, HKCR has been conducting Fellowship Examination and Exit Assessment for its three specialties. For Clinical Radiology and Clinical Oncology, the Fellowship examinations are organized jointly with The Royal College of Radiologists (RCR) of the United Kingdom, an arrangement taken to maintain standards and stay on par with international radiological qualification. The HKCR devises general training regulations that apply to all three specialties and establishes a specific training curriculum and examination format for each specialty. Accreditation visits to training centers are also periodically arranged by the HKCR to assess and approve the training centers and training programs.

The training program for each specialty of radiology consists of two parts: Basic Training and Higher Training. Basic Training covers the initial 4 years of post-graduate training. During this period, a radiology trainee is expected to pass the first (part I) and final (part IIA and B) examinations.

These examinations are held in different months of the year, and the dates of the examinations are announced at least 3 months ahead of time. The Part IIB Examination is conducted in the form of a Joint Fellowship Examination, in which a board of examiners consisting of overseas and local examiners is formed in each sitting to conduct the examination in Hong Kong. The Higher Training covers 2 years and comprises in-depth training in general radiology, subspecialty training in radiology, and management training including audit and quality assurance. The Higher Training must be completed within 4 years after passing the Part IIB Fellowship Examination in Radiology or Clinical Oncology.

Before completion of the entire training, a trainee must pass the Exit Assessment. The Exit Assessment is 
held half-yearly with the objective to evaluate the overall adequacy of training and competence of trainees prior to admission as Fellows of the HKCR. Several important requirements have to be fulfilled before a trainee is qualified to sit for the exit assessment. First, the trainee has to pass both parts of the College Fellowship Examinations and have completed not less than 6 years of full-time accredited training, including the required period of accredited clinical experience outside the mother discipline and not less than 2 years of higher training. Second, the trainee must become a Member of the College for at least 1 year prior to the Exit Assessment. In addition, the trainee must have attended the training courses organized by the College. The College has stringent principles in encouraging trainees to attend all the training courses, and a trainee will be allowed to sit for Exit Assessment only if he or she has obtained a certificate of satisfactory attendance of at least $80 \%$ of the sessions for every training course including all mandatory lectures. If a trainee fails to achieve this minimum requirement for a training course, he or she must make up for the sessions of absence within the remaining training period. In the Exit Assessment, a panel of assessors scrutinizes the profile of the trainee to review whether he or she has received comprehensive training. The assessment includes reviews of records of training, documented in the personalized training logbook, audit projects, publications and presentations, regular appraisal reports by supervisors, and the final appraisal report by the Training Head.

An interview of the trainee is also conducted to assess different facets pertinent to the overall training, including professional maturity and attitude, appropriateness of independent practice, and contribution to professional activities. A maximum of three attempts is allowed for the Exit Assessment, all of which must be completed within 3 years from the time that the candidate becomes eligible to apply for the Exit Assessment. If the candidate fails all three attempts, he or she needs to undergo the entire Higher Training all over again. Passing the Exit Assessment is a landmark for completion of training, and the trainee is then qualified to be awarded the Fellowship of the Hong Kong College of Radiologists (FHKCR). When a trainee does not pass the Exit Assessment, the head of the respective training department will be informed of the Panel's comments on the weaknesses of the trainee and advice concerning appropriate remedial actions. The entire training program and board examination are summed up in Fig. 4.

To remain in the Specialist Register after successfully passing the Exit Assessment, radiologists need to fulfill the required CME/CPD credit points. These are Continuing Medical Education (CME) and Continuous Professional Development (CPD) activities, including training courses, seminars, and congresses for radiology updates and introduction to cutting-edge technology.

The structured residency training program in approved Radiology Training centers in Hong Kong ensures quality, standards, and efficiency. In addition, the training must keep pace with modern healthcare demands and development according to community needs. The Hong Kong College of Radiology continues to embark on liaisons with and support from local and international bodies, which are essential partners for driving progress. Continuous negotiation with the government and Administration and Financing Authorities is also made to uphold training standards, improve training conditions, and gain training resources including career development and installation of cutting-edge equipment for training.

\section{Residency training program and board examination in Korea}

In Korea, there are two different tracts to becoming a medical doctor: the pre-medical and college of medicine system and the medical graduate school system. All medical students have to choose one of these two tracts. If high school students enter the first tract, they can finish a 2-year pre-med course and a 4-year medical college course continuously. The other tract leads to a 4-year bachelor's degree, and candidates will have to take an entrance examination to qualify for the medical graduate school program. Both tracts have the same curriculum.

Only after 3 years of military service can candidates start their residency training, which takes 4-5 years to complete before becoming a specialized physician. In radiology, they have to complete a 1-year internship and a 4-year residency before they become eligible to take the government-accredited board certification examination. A 1-2-year fellowship is required to earn a subspecialty. Figure 5 summarizes the

\begin{tabular}{|c|c|c|}
\hline Clinical Year & Basic / Intermediate Training & Higher Training \\
\hline 1 Year & 3 Years & 2 Years \\
\hline Part I \& Part IIA Exam & Joint (Part II B) Exam \\
\hline
\end{tabular}

Fig. 4 Summary of Hong Kong's residency training program and board examination 


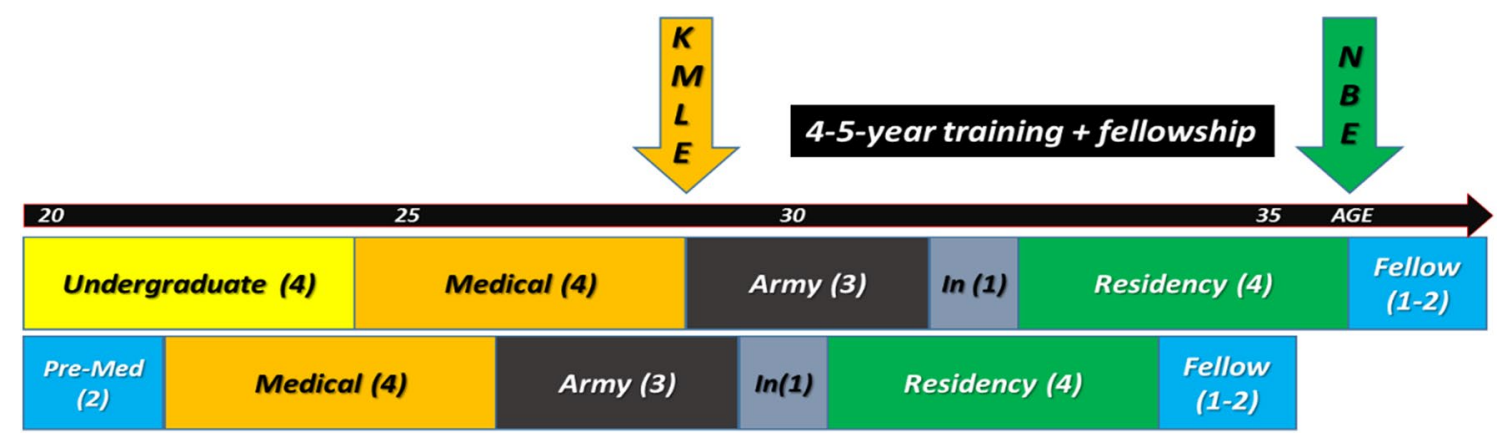

Fig. 5 Outline of the residency training timeline in Korea

duration and composition of the residency training program in Korea.

Radiology is the most popular discipline in Korea. Quotas for radiology residents are determined by the KSR, KHA, and the government. Every hospital selects residents based on their own criteria. Training hospitals pay the residents a salary of about $\$ 30,000 /$ year. No funding comes from the government.

The quota of radiology residents has decreased annually because of the government's policy of increasing the number of primary care physicians and decreasing that of specialists. Annual accreditation of training hospitals is decided based on the number of supervising radiologists, facilities, academic activities, etc.

Evaluation programs for residents are the annual in-training programs and training records, which are certified by the KSR annually. The proportion of questions in in-training examinations is $10 \%$ on pure physics and $90 \%$ on the subjects of nine subspecialties covering applied physics, applied anatomy, and clinical questions.

Residents must fulfill some requirements to take the board certification examination. They must rotate around nine subspecialties on a monthly basis for 4 years including 2 months in nuclear medicine, although nuclear medicine is a different department in Korea.

Residents have to participate in more than 30 domestic or international conferences and publish more than one original article or two case reports on scientific journals listed in SCI (Science Citation Index) and SCIE (Science Citation Index Expanded) and some other Korean journals related to radiology. A scientific paper is required to be eligible for the board examination.

Board certification is accredited by the government. The board certification examination is taken at the end of the 4-year training period, usually twice for the first and second parts of the examination, in January. The interval between the two is usually 1 week. Only residents who already passed the first part of the examination can advance to the second. The pass rate is $90-97 \%$.
The first part of the examination is composed partly of printed image photos (55-60\%) and partly without printed image photos (40-45\%). The second part is a slide (PowerPoint slides) test using a beam projector and image interpretation with printed image photos. For the first time last year, in 2016, a mobile device, a tablet, was introduced in the 2017 examination. It was used as an alternative to printed image photos and PowerPoint slide images in both the first and second part of the examinations.

Using the tablet, examinees can control image size (zoom and pan), brightness, and contrast. It overcomes the image number and quality and can show multimedia items. It also has a stack mode item similar to PACS or picture archiving and communication system.

\section{Residency training program and board examination in Mongolia}

Radiology education in Mongolia has three stages. The first is undergraduate education in medicine, the second is graduate education (residency training), and the last is postgraduate education (subspecialty training).

The undergraduate course or bachelor's degree consists of a 2-year pre-medical course and 4-year medicine course. The first 2 years of the medicine course cover basic medical sciences, one of which is Radiology. The last 2 years are spent on clinical clerkships, which also include Radiology.

The residency training course follows after 2 years of internship. The length of residency training has been extended from 1.5 to 2 years since 2010 when a nationwide core curriculum for residency training was implemented.

It was not until 2000 that the Mongolian Board of Radiology was introduced, and the first national examination was conducted in 2001 for Radiology Specialties and Subspecialties. It covers disciplines in Diagnostic Radiology, Interventional Radiology, and Radiation Oncology. In 2006, certificates of additional qualification in Interventional Radiology and Pediatric Radiology were added as sub-specializations. 
The challenges of the residency training program in Mongolia are the duration of the training program itself, which is only 2 years, poor quality of training in some universities with no accreditation, limited budget for investment in teaching materials for residents (Interventional Radiology), limited resources for quality textbooks in the Mongolian language, the rapid increase in the number of Radiology residents without any control or surveys by the Ministry of Health and Health Development Center of Mongolia, limited range of well-educated human resources, and non-accreditation of the curriculum of residency training since 2010.

To address these challenges, the Mongolian Society of Radiology is extending the length of residency training to 3 years starting in 2018 . The curriculum of residency training will be updated to be accredited on par with international universities. Efforts in improving inter-society cooperation in the field of residency training through exchange programs and an Asian standard curriculum are also underway. Increasing the number of fully trained, well-educated teachers and decreasing the number of training centers will also improve the current system.

\section{Residency training program and board examination in Myanmar}

At present, Myanmar has a residency training course in only four medical universities, namely University of Medicine 1, University of Medicine 2, University of Medicine (Mandalay), and Defense Services Medical Academy.

About 20 candidates are selected for the residency training course at each university every year. A 3-year course is conducted for those who are selected by the selection examination.

To be eligible, one must have been a government servant medical doctor for at least 1 year in government service, have written two papers, pass theory (subject matter) tests 1 and 2 covering General Medicine, Surgery, Obstetricsgynecology, and Pediatrics, pass an oral examination, and pass the test for English proficiency. The oral examination is for those who pass the theory examinations.

The 3-year course of residency is divided into two parts: Part 1 (9 months) and Part 2 (27 months). Part 1 covers subjects on Medical Imaging Physics, Imaging Technique, Imaging Anatomy, and Emergency Radiology. After the 9-month period, examinations are conducted for theory tests [multiple-choice question (MCQ) and multiple short questions (MSQ)] and an oral examination. Candidates need to pass the Part 1 examination to advance to Part 2. Two supplementary examinations are held at 3-month interval.

Part 2 includes a protocol for dissertation, and this must be submitted to the Protocol Board within the first 6 months. The dissertation must be submitted to the Board of Examiners at least 3 months before the final examination. The curriculum covers subjects on diagnostic radiology of the respiratory system, CVS (cardiovascular system), CNS (central nervous system), head and neck, the musculoskeletal system, GI (gastrointestinal) and liver, eye and ENT, pediatrics, women's imaging, breast imaging, and congenital disorders.

Attending multi-disciplinary conferences, journal reading, case presentations, and topic discussions are additional requirements in Part 2 as well as participating in basic interventional radiology, CT, MRI, ultrasonography, mammography, plain and contrast examination procedures, and X-ray film reading.

For the assessment, a formative type of examination is performed at the end of each system training. After completion of the Part 2 course, a final examination is administered by the Examination Board, composed of a Co-Examiner and External Examiner, Professors, Associate Professors, and Lecturers from all four universities. The examination is conducted at only one university every year. All four universities take turns in hosting the examination.

The final examination has three compartments. Compartment 1 consists of a theory paper for a total of 100 marks: $50 \%$ multiple choice questions and $50 \%$ multiple short questions. Compartment 2 is a practical examination for a total of 100 marks: $80 \%$ case scenarios and $20 \%$ rapid film reading. Compartment 3 consists of a viva voce for a total of 100 marks. A passing mark is $60 \%$ for each compartment.

A supplementary examination is held at a 6-month interval. Candidates are given two chances to pass the supplementary examination or they will be terminated from the training course.

\section{Residency training program and board examination in Nepal}

To be enrolled as a Radiology MD in Nepal, one has to have completed an MBBS (Bachelor of Medicine, Bachelor of Surgery) degree and pass the medical entrance examination conducted by different institutions, both public and private. Usually, the best performers on the medical entrance examination in Nepal choose to have a residency in Radiology.

The duration of the MD Residency is 3 years. A 200-point written examination is administered at the end of the first year, and a 200-point oral examination is conducted at the end of the final year.

Residents rotate in different modalities such as x-ray reporting, mammograms, USG (ultrasonography), CT scans, MR imaging, and special procedures for training. This rotation starts in the first year and lasts until the final year. There is a 24-h posting in the emergency department to perform USG and interpret X-rays and CT scans, etc. 
In the second year, a thesis proposal must be approved by Institutional Review Board (IRB). Upon the approval of the thesis, data collection is started and must be completed and submitted to the examination section within a year.

Seminars included in the whole syllabus must be attended by residents, and residents must participate in clinical rounds in different departments.

Completion, submission, and approval of the thesis are all due in the final year. Without an approved thesis, a resident is not allowed to sit for the final examination. Examiners consist of local and international senior faculties.

The examination is conducted by the Nepal Medical Council twice a year. It includes multiple-choice questions, short questions, and clinical cases and is all in written form. Accreditation by the NMC is necessary to practice Radiology in Nepal.

The entire residency training program is intensive and provides residents enough exposure to become a general radiologist, though the time period may be too short considering the increasing number of modalities.

In an effort to address this area for improvement, the Nepal Radiological Society, in collaboration with the government, is working on extending the duration of residency from 3 to 5 years. Fellowships are also necessary to establish subspecialty training programs.

\section{Residency training program and board examination in Pakistan}

In Pakistan, many government and private medical colleges need approval from the Pakistan Medical and Dental Council (PM\&DC) before they start operating. Primary Medical Education is for 5 years followed by a 1-year internship in a PMDCrecognized university, public or private hospital. PMDC serves as the regulatory authority where, after the internship, doctors register. They are issued their unique Medical License number and are called Registered Medical Practitioners.

There are two separate residency programs in Pakistan, which are run by separate institutions. The first is the College of Physicians and Surgeons Pakistan (CPSP), operating through their Regional Centers in all the big cities. After attaining a degree, degree holders are called Fellows of the CPSP, written as FCPS (a specialty like Medicine or Diagnostic Radiology). The second is MD (Doctor of Medicine) / MS (Master of Science)/MDS (Master of Dental Surgery) / DIPLOMA Programs run by individual universities.

The CPSP offers two kinds of programs: Fellowship, written as FCPS, is a 4-year training program recognized by the PMDC as a Class III Specialty and Membership, written as MCPS, is a 2-year training program.

After completion of a 1-year internship, candidates are eligible to take the CPSP entrance test of their specialty of interest, called the FCPS Part I examination in different subjects. Currently, there are more than 40 disciplines in which one can take a FCPS Part I Examination.

After passing the FCPS Part I examination, candidates can apply to PMDC/CPSP-recognized hospitals for training. In the first 2 years, residents are trained in their respective fields and do a compulsory rotation according to the specialty they have chosen. Then they attend compulsory workshops on dissertation writing and internet skills to become eligible for the Intermediate Module (IMM).

The IMM consists of a written test followed by a practi$\mathrm{cal} /$ oral examination. The residents have to pass this examination and, in addition, select a dissertation topic on which they have to do research. They have to submit the dissertation to be eligible to sit for the FCPS Part 2 Examination.

After 4 years of vigorous training and fulfilling the criteria to sit for the Part 2 Examination, residents then have to undergo a Theory Examination and Viva/Practical, after which they are awarded the Fellowship Degree (FCPS) and are considered specialists in their respective fields.

For the residency training run by universities affiliated with PMDC-approved teaching hospitals and managed by the syndicate of the universities, residents may take diploma courses and MD/MS/MDS. Diploma courses (DMRD) are 2-year training programs and start after clearing the entrance examination at a specific university followed by an exit examination after completion of the required training (2 years) and submission of the thesis. Each university has its own specific pattern of examination. MD/MS/MDS, on the other hand, are 4-year training programs started after the candidates clear the entrance test (at a specific university). After 2 years of training, residents have to clear the Intermediate Module Examination (IMM) followed by thesis submission within the stipulated time period, after which the resident is eligible to take the exit examination (Part 2 Examination). PMDC approves the candidates as Class III Specialists upon passing the exit examination for the MD/ MS.

Due to continuous turnover and increase in medical graduates every year, the slots for training are lacking with many residents having to wait for the next induction process. Each supervisor is allowed to supervise a maximum of seven to nine residents depending on the availability of supervisors in each hospital. This results in difficulty for residents. There are only a few training facilities, and sometimes residents have to move to another city to find an affiliation with a hospital or training program.

The Radiological Society of Pakistan (RSP) has always helped the radiologist community in the country since its establishment in 1986. Regular CME (Continuing Medical Education)/workshops are held in various cities in the country under the umbrella of the Radiological Society of Pakistan, which have been very beneficial for the country's 
budding radiologists. The RSP regularly holds its Annual Radiology Conference where a large Scientific Meeting is organized and attended by many national and international radiologists who present their latest work and research. Each year at the conference, the Best Paper Presenter and Best Poster Presenter are awarded to residents to encourage their participation. In addition, they are also provided educational grants upon the recommendation of the Educational Committee and approval of the President of the RSP.

The International Liaison Committee of the RSP has been very active since its formation and has been successful in signing memorandum of understanding (MoU) with our sister societies, such as the Turkish Society of Radiology (TSR), European Society of Radiologist (ESR), Korean Society of Radiologist (KSR), and Iranian Society of Radiologist (ISR). Their speakers are also invited to the annual conferences to present their work. As a result of the combined efforts of the RSP and ESR, the European Board of Radiology has agreed to organize an international examination called the European Diploma in Radiology (EDiR) in Islamabad, Pakistan, on 21 September 2017. The registration for the examination is already full and is expected to be a successful event.

\section{Residency training program and board examination in the Philippines}

The Radiology residency in the Philippines is a 4-year program and involves modality-based rotation to gain exposure to different fields such as general radiology, mammography, ultrasound, computed tomography, interventional radiology, and magnetic resonance imaging.

Prior to taking the diploma examinations, residents are required to cover certain numbers of different cases in various fields as shown in Table 3.

Other requirements include attendance at scientific meetings, updated log books, in-service examinations, research papers, endorsement letters from the training officers, photocopies of hospital accreditation when residency training started and an updated one, attendance at departmental

Table 3 Number of cases required in different fields of radiology

\begin{tabular}{lcccr}
\hline & $\begin{array}{l}\text { General radi- } \\
\text { ology }\end{array}$ & UTZ & CT & MRI \\
\hline Neuro, H and N & 480 & 360 & 375 & 300 \\
Pulmonary, cardio & 5000 & 120 & 300 & 40 \\
GI, GU & 1000 & 500 & 400 & 150 \\
MSK & 1000 & 50 & 100 & 100 \\
Breast & 100 & 300 & - & 10 \\
OB-GYN & 10 & 300 & 100 & 20 \\
Pediatric & 700 & 450 & 200 & 150 \\
\hline
\end{tabular}

and interdepartmental conferences, and basic life support training.

The Philippine College of Radiology (PCR) has an Accreditation Committee that visits the various hospitals throughout the country to check on the status of each accredited Training Program in Radiology. This is done every 3 years for each hospital. The Residency Training Council (RTC) is composed of training officers from all accredited hospitals in the country. Currently, there are around 40 accredited hospitals.

The RTC oversees and regulates the training programs, which are applied to all institutions. It administers bimonthly in-service examinations for all residents in training (total of ten examinations at 100 items per examination). It also conducts bimonthly scientific meetings/forums for residents. Research opportunities are provided by the RTC for each resident as well.

The board examination is given by the Philippine Board of Radiology to residents and confers the title of diplomate upon successful completion of these examinations. The examination is divided into three parts. Examination I covers the subjects of physics, radiation protection, radiological procedures and anatomy and is a prerequisite to Examination II, which is a written examination on pulmonary, cardiovascular, genitourinary, women's, gastrointestinal, musculoskeletal, neuroradiology, and head and neck imaging. The final part, Examination III, is a practical and oral examination.

Each examination consists of 100 items. A passing score is $75 \%$ of the mean passing score or one standard deviation from the mean, and the passing average should be no less than 50\% (60-65 score). If an examinee fails, at most three subjects can be taken again for the following year. If an examinee fails more than three subjects, he or she will have to take the entire examination all over again.

Once a candidate has passed the various examinations given by the Philippine Board of Radiology, he/she is called a Diplomate of the Philippine Board of Radiology. Then, after at least 1 year, he or she can submit a Research Paper to the Research Committee of the Philippine College of Radiology; if accepted by the Committee, she or he can then apply as a Fellow of the Philippine College of Radiology. If no ethical complaints are raised against the candidate, the PCR will then bestow the title of Fellow of the Philippine College of Radiology.

To inspire enthusiasm among residents, they are encouraged to join the Battle of the Brains where 28-32 teams of residents from different chapters of the society compete. The finals take place during the pre-congress of the annual convention of the society. The Best Presenter is also awarded to residents who take part in interesting case presentations.

Subspecialty training in the Philippines is still modalitybased (e.g., ultrasound, CT, MRI, etc.), but not system-based (e.g., Neuroradiology, MSK Radiology, GIT Radiology, etc.) 
or based on a special field of study (e.g., Pediatric Radiology, Oncologic Radiology, Women's Imaging, etc.). This is clearly an area of opportunity for the residency training in the Philippines.

\section{Residency training program and board examination in Thailand}

The Royal College of Radiologists of Thailand (RCRT) is the main committee for training for all radiological curricula, and each curriculum has a training subcommittee. The RCRT has roles in providing and implementing the training policies, regulating and supervising the training institutes, and administrating the Board examination. There are seven curricula in the field of Radiology, as shown in Table 4: three for residency programs and four for subspecialty programs. The three residency programs consist of Diagnostic Radiology, Radiation Oncology, and Nuclear Medicine. Each is a 3-year training program. The subspecialty programs include Advanced Diagnostic Body Imaging, Diagnostic Neuroimaging, Body Interventional Radiology, and Interventional Neuroradiology. Each subspecialty is a 2-year training program.

The first-year residents in all residency programs must complete Radiation Physics and Radiobiology courses and must take the examination provided at the end of the first year.

The Diagnostic Radiology program includes 29-month rotation in Diagnostic Radiology, 2-month rotation in Radiotherapy, 2-month rotation in Nuclear Medicine, 1-month rotation in Pathology, and 2-month rotation for elective subjects. Professionalism, attitude, morals and ethics, communication skills, scientific knowledge of medicine and radiology, patient care, continuous professional development, and leadership are also integrated. The educational tests and measurements comprising written and oral examinations at the end of year III are targeted to assess both basic and comprehensive knowledge gained from the training program.

Research is also a compulsory part of each training program.
The 3-year duration of the residency training program may be too short, but good collaboration among radiological societies and all eight training centers in Thailand makes the residency training program systematic. Moreover, the RCRT actively takes part in international collaborations and societies for education and research.

\section{Residency training program and board examination in Singapore}

The residency program in Singapore involves full or conditional registration with the Singapore Medical Council with a continuum of 5 years. It comprises Neuroradiology, Musculoskeletal Radiology, Breast Imaging, Body Imaging, Interventional Radiology, Pediatric Imaging, Ultrasound, Nuclear Medicine, and General Radiology. There are postgraduate examinations and qualifications for the Fellowship of the Royal College of Radiologists (FRCR).

The first year of the residency program is considered a transitional year, and during this time, residents are introduced to general Radiology, ultrasound, and cross-sectional imaging. In addition to general Radiology, residents start taking up subjects in Nuclear Medicine, CT, ultrasound, and MRI in their second year, after which they have to sit for the FRCR Part 1 examination.

Rotations through radiological subspecialties happen in the third and fourth year, and residents have to take the FRCR 2A examinations. After consolidation and selection of subspecialties in the 5th year, they have to sit for the FRCR 2B or Masters of Medicine Radiology examinations.

Two weekly electronic-based tutorials with individual grades and meetings with supervisors and monthly consultation following the FRCR 2B oral examinations are conducted. The program involves multiple clinical-radiology rounds with the clinical specialty departments. Reference materials in StatDx and e-anatomy are electronic. Resident's radiology reports are audited on a regular basis, and feedback is given. Research opportunities are provided, and residents are encouraged to participate. However, since

Table 4 Thailand's seven curriculum in radiology

\begin{tabular}{ll}
\hline $\begin{array}{l}\text { Specialty: } 3 \text { years } \\
\text { Residency training in Diagnostic Radiology }\end{array}$ & $\begin{array}{l}\text { Diploma of the Thai Board of Diagnostic Radiology } \\
\text { Diploma of the Thai Board of Therapeutic Radiology and Oncology } \\
\text { Diploma of the Thai Board of Nuclear Medicine }\end{array}$ \\
$\begin{array}{l}\text { Residency training in Nuclear Medicine } \\
\text { Subspecialty: } 2 \text { years }\end{array}$ & $\begin{array}{l}\text { Diploma of the Thai Subspecialty Board of Advanced Diagnostic Body Imaging } \\
\text { Fellowship training in Advanced Diagnostic Body Imaging } \\
\text { Fellowship training in Body Interventional Radiology }\end{array}$ \\
$\begin{array}{l}\text { Fellowship training in Diagnostic Neuroimaging } \\
\text { Fellowship training in Interventional Neuroradiology }\end{array}$ & $\begin{array}{l}\text { Diploma, Thai Subspecialty Board of Diagnostic Neuroimaging } \\
\text { Diploma of the Thai Subspecialty Board of Interventional Neuroradiology }\end{array}$ \\
\hline
\end{tabular}


promotion to the next level is dependent on clearing relevant examinations, emphasis is placed on teaching and passing such examinations.

The structured residency training program in Singapore ensures consistency across different hospitals. However, "real training" does not start until after passing postgraduate examinations, and this may cause a delay in promotions and advancement if a resident fails an examination.

\section{Residency training program and board examination in Sri Lanka}

The residency training program in Sri Lanka commenced in 1981, and in 2016, subspecialties were introduced.

The training program is conducted by the Board of Study in Radiology, Postgraduate Institute of Medicine (PGIM) University of Colombo, Sri Lanka, and is funded by the Ministry of Health. Entry into the training program is through a competitive Selection Examination, which is conducted by the PGIM each year. The number of training slots is predetermined each year by the Board of Study in Radiology and PGIM in consultation with the Ministry of Health.

The training program for General Radiology consists of six stages spanning over 60 months: 36 months of pre-MD training and 24 months of post-MD training. An apprenticeship type in-service training program is carried out at accredited training centers under the supervision of recognized trainers.

Lectures and film viewing sessions, too, are organized during the pre-MD training period. Periodical appraisals of a trainee's progress are made throughout the program. Formal assessments by the MD Radiology Part I and MD Radiology Part II examinations are done at the end of year 1 and year 3 , respectively.

Residents who pass the MD Radiology examination proceed to post-MD training, which is arranged in Sri Lanka as well as overseas. Overseas training is provided so the residents can gain further experience in well-equipped centers of excellence. Post-MD training for subspecialties differs from and is longer than that for General Radiology.

At the end of post-MD training, residents have to pass the Pre-Board Certification Assessment consisting of summative assessment of the resident's portfolio (evaluated by two radiologists and one examiner from another discipline) and an oral examination.

Upon successful completion of training, the resident is awarded a postgraduate degree in MD Radiology and Board Certification as a specialist in General Radiology or in a particular subspecialty in Radiology.

Overall, the residency training program of Sri Lanka is very systematic. The challenges that need to be addressed are the lack of expensive radiology equipment at accredited training centers and the non-availability of trainers for the subspecialty training.

The Sri Lanka College of Radiologists (SLCR) supports the training program and continues its efforts to coordinate with the Ministry of Health to obtain adequate radiologists and equipment in hospitals. Educational programs such as lectures, clinical meetings, workshops, and conferences to update the knowledge of radiologists and trainees are also arranged by SLCR.

\section{Residency training program and board examination in Japan}

The special training in Radiology in Japan consists of twostep training and certification. The first step is a 3-year training and board examination covering Diagnostic Radiology and Radiation Oncology. To become a diagnostic radiologist or radiation oncologist, trainees undergo an additional 2-year training and take the second step, a board examination. Nuclear medicine is included in Diagnostic Radiology.

All resident trainings are performed at official facilities authorized by the Japan Radiological Society. Training facilities are classified into core teaching hospitals, cooperative hospitals, and specialty-focused facilities. Most core teaching hospitals are university hospitals and large general hospitals. Specialty-focused facilities include imaging centers and children's hospitals. Several facilities construct networks centered upon core teaching hospitals, and trainees rotate within these networks. The numbers of core teaching hospitals and cooperative hospitals are about 200 and 500, respectively, and that of the specialty-focused facility is 20 .

The required contents for certified radiologists in the first training include Diagnostic Radiology [plain X-ray, CT, MRI, US, AG• IVR (angiography interventional radiology), and GI], Nuclear Medicine, and Radiation Oncology. Table 5 outlines these examinations and the number of cases required. Cases encountered at conferences or at official e-learning courses can be included.

To complete the second training and take the board examination for diagnostic radiologists, there are considerable additions to the number of cases in each examination.

The first board examination, the written examination, consists of 105 multiple-choice questions including 15 basics, 55 Diagnostic Radiology, 15 Nuclear Medicine, and 20 Radiation Oncology. The test duration is $2 \mathrm{~h}$ and $30 \mathrm{~min}$. The passing score is about $60-65 \%$. The pass rate is about 85-90\%.

The second board examination consists of two partswritten and oral. The written examination consists of 80 multiple-choice questions including 5 basics and 75 case review (Diagnostic Radiology 60, Nuclear Medicine 15) (Figs. 6, 7). 
Table 5 Required contents for certified radiologists in the first training

\begin{tabular}{lll}
\hline Fields & Examinations & $\begin{array}{l}\text { Number } \\
\text { of guides }\end{array}$ \\
\hline Diagnostic Radiology & Plain X-ray & 400 \\
& CT & 600 \\
& MRI & 300 \\
& US & 120 \\
& AG IVR & 30 \\
Nuclear Medicine & GI & 60 \\
Radiation Oncology & & 50 \\
\hline
\end{tabular}

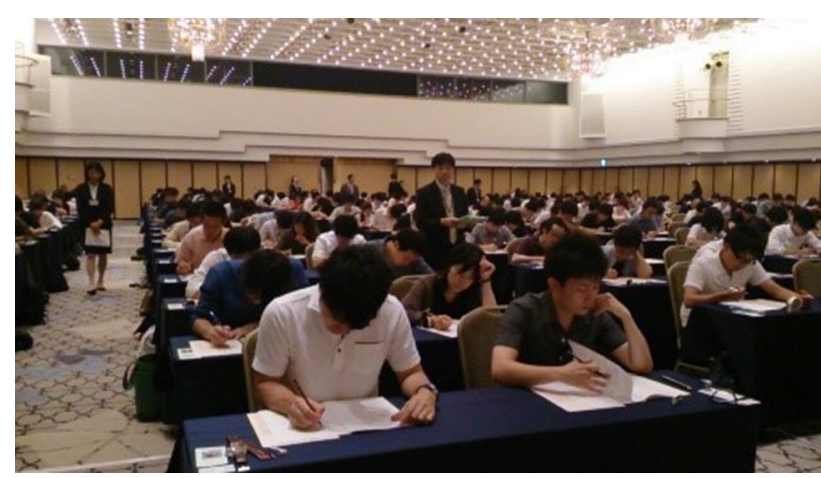

Fig. 6 Photo taken during the written examination for Diagnostic Radiology, which was administered last August. This examination is held at a hotel every year

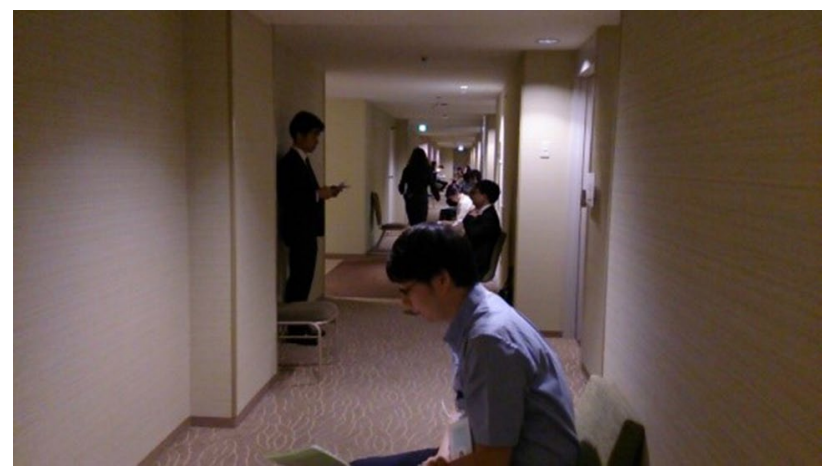

Fig. 7 Examinees wait outside the room for their turn for their oral examination

The oral examination is performed 1 day after the written one. It consists of four sections, 3 of which are on Diagnostic Radiology and one on Nuclear Medicine. Examinees have to rotate around four rooms where an examiner for each specialty in these fields is situated and take an oral examination using a viewer. Each section has three to four cases to be completed within $15 \mathrm{~min}$. The passing score is above $60 \%$ in both the written and oral examinations. The pass rate is about $80-85 \%$.

The maintenance of board certification does not require any more examinations. The requirements, however, are screening of applicant materials, participation in major JRS congresses, participation in compulsory lectures, and achievement of publications and are to be completed every 5 years.

The Japanese medical board certification system in all medical fields is going to change. To this day, each society administers each specialty certificate independently. In 2014, a new national board, the Japanese Medical Specialty Board (JMSB), was established to authorize training programs and facilitate initial certification and maintenance. This new medical board certification system will start in 2018 .

An outline of the new training system has been constructed; however, the details are left to the discretion of each society. Maintenance of certificates will be stricter under the regulations of the new national board.

Acknowledgements This paper was written based on the presentations at the Asian Radiology Summit 2017 held during the JRC 2017 by the following representatives of the participating societies (in order of presentation): Khalilur Rahman, MD (Bangladesh Society of Radiology and Imaging), Yeun-Chung Chang, MD (Chinese Taipei Society of Radiology), Lilian Leong, MD (Hong Kong College of Radiologists), Won Jae Lee, MD (Korean Society of Radiology), Dagvasumberel Gonchigsuren, MD (Mongolian Radiological Society), Khin Hla, MD (Myanmar Radiological Society), Ansari Mukhtar Alam, MD (Nepal Radiologist Association), Khawaja Khurshid Ahmad, MD (Radiological Society of Pakistan), Jerome A. Gaerlan, MD (Philippine College of Radiology), Juntima Euathrongchit, MD (Radiological Society of Thailand), Andrew Tan, MD (Singapore), Shanthini Rosairo, MD (Sri Lanka College of Radiologists), and Noriyuki Tomiyama, MD, PhD (Japan Radiological Society). We thank Miss Liza Mia Christine F. Maquiraya for editing this manuscript. 